\title{
Physician preferences for management of patients with heart failure and arrhythmia
}

\author{
Ümit Yaşar Sinan a,* , Mehdi Zoghi ${ }^{\text {b }}$, on behalf of CAS-TR Group ${ }^{1}$ \\ a Istanbul University Institute of Cardiology, Department of Cardiology, Turkey \\ b Ege University School of Medicine, Department of Cardiology, Turkey
}

\section{A R T I C L E I N F O}

Article history:

Received 14 May 2017

Received in revised form 7 June 2017

Accepted 8 June 2017

Available online 8 June 2017

\begin{abstract}
A B S T R A C T
Heart failure (HF) is one of the most important causes of morbidity and mortality in the world. It is a progressive and irreversible disorder. Survey and questionnaires are helpful verify that real-life daily practice is keeping with what is recommended in the guidelines. In this questionnaire which is consisted of 32 questions about current diagnostic and therapeutic strategies of HF and arrhythmia, we aimed to evaluate awareness of physicians who are interested in HF and arrhythmias about current diagnostic and therapeutic strategies of HF.

This survey was conducted in university hospitals, state hospitals, training and research hospitals, private hospitals and medical centers in seven geographical region of Turkey between January and February 2017. The study groups were consisted of 177 physicians (163 cardiologists, 7 cardiovascular surgeons, 6 internal medicine physicians and 1 primary care physician). Less than half of the physicians ( $\mathrm{N}: 71,40.1 \%)$ have used natriuretic peptides in the diagnosis of HF and 76.3\% of physicians were aware of HF with mid-range EF (HFmrEF)-patients with HF and a left ventricular ejection fraction (LVEF) that ranges from 40 to $49 \%$. Despite optimal medical therapy with diuretic, ACEIs, ARBs, BB and mineralocorticoid receptor antagonists (MRAs), the ratio of physicians who have $>50 \%$ symptomatic patient population were $6.8 \%$. In this patient, angiotensin receptor neprilysin inhibitor (ARNI) was the treatment choice among $62.7 \%$ of physicians. Interestingly, $10.2 \%$ of physicians have never heard before ARNI. While only $13.6 \%$ physicians had $>30 \%$ patients with HFpEF, most of the physicians (30.5\%) had between $21 \%$ and $30 \%$ patients with HFpEF.

In this questionnaire we want to see a picture from daily practice of physicians who are interested in heart failure. We aimed to measure awareness about current European Society of Cardiology (ESC) heart failure guideline recommendations, new classification of HF according to LVEF and new diagnostic and therapeutic improvements. (C) 2017 The Society of Cardiovascular Academy. Production and hosting by This is an open access article under the CC BY-NC-ND license (http://creativecommons.org/licenses/by-nc-nd/4.0/).
\end{abstract}

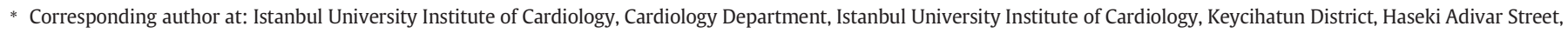
Fatih, Istanbul, Turkey.

E-mail address: umit.sinan@istanbul.edu.tr (Ü.Y. Sinan).

1 Gülay Gök, MD, Haşim Mutlu, MD, Arzu Neslihan Akgün, MD, Özlem Yıldırımtürk, MD, Veli Polat, MD, Şerife Ayşen Helvacı, MD, Mevlüt Demir, MD, Emine Gazi, MD, Gönül Açıssarı,

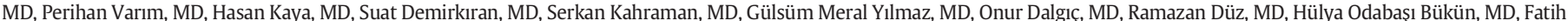

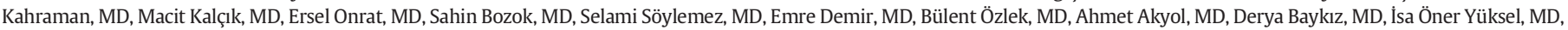

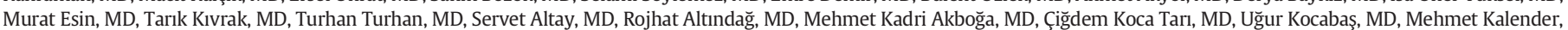

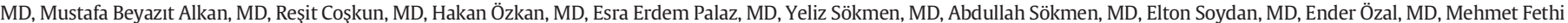

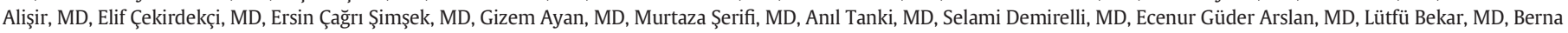

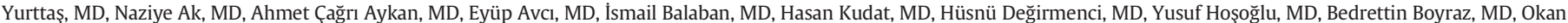

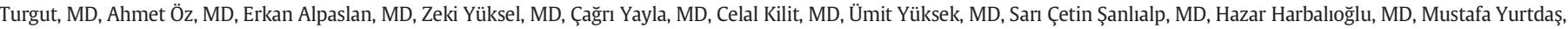

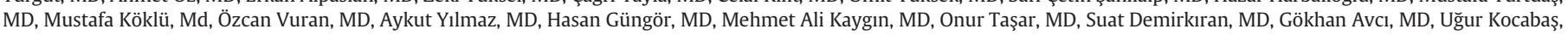

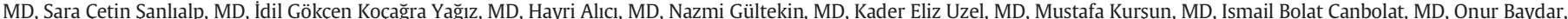

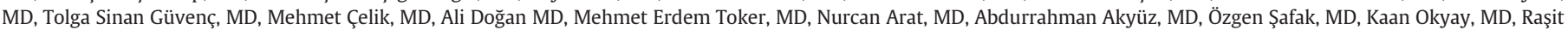

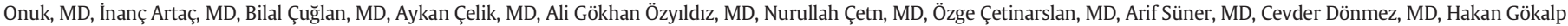

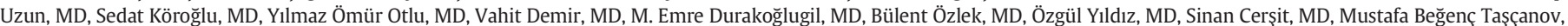

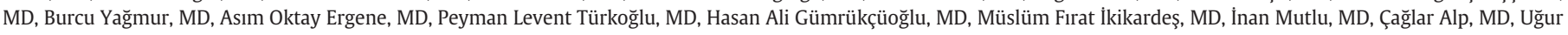

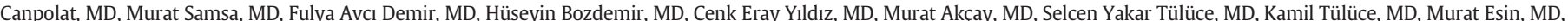

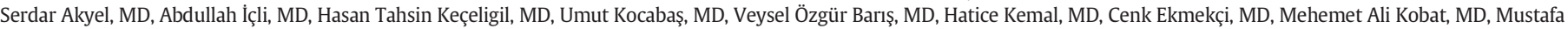

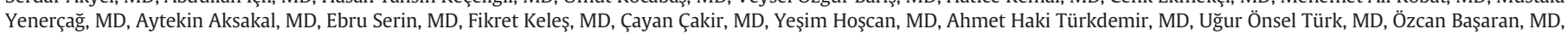

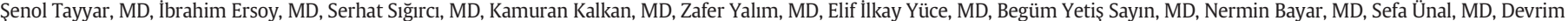

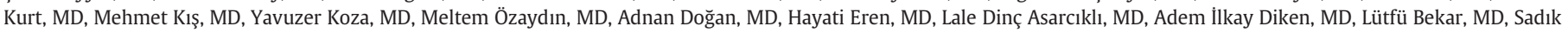
Volkan Emren, MD, Hazar Harbalıŏlu, MD, Oğuzhan Çelik, MD, Yusuf Çekici, MD, Tolga Aksu, MD, Fatih Aytemiz, MD, Barış, Buğan, MD.
}

Peer review under responsibility of The Society of Cardiovascular Academy. 


\section{Introduction}

Heart failure (HF) is one of the most important causes of morbidity and mortality in the world. ${ }^{1}$ It is a big burden to healthcare economy. The prevalence is 0.4 to $2 \%$ in general European population ${ }^{2}$ and 5 million Americans with chronic HF are mostly attributable to inpatient hospitalization. ${ }^{3}$ Currently approximately 26 million adults live with HF in the world ant this number is substantially increasing with aging population. The prevalence is not clearly known in Turkey, but in the HAPPY study it was estimated as $2.9 \%$ in adults. ${ }^{4}$

Heart failure is a progressive and irreversible disorder. Therefore, prevention of HF is of great importance. At first, it is required to control risk factors of $\mathrm{HF}$ and leading underlying causes and then if the disease has occurred, the guidelines recommended therapy should be implemented. Survey and questionnaires are helpful verify that real-life daily practice is keeping with what is recommended in the guidelines. Health professionals are encouraged to take the guidelines fully into account when exercising their clinical judgement, as well as in the determination and implementation of preventive, diagnostic or therapeutic medical strategies.

In this questionnaire we aimed to evaluate awareness of physicians who are interested in HF and arrhythmias about current diagnostic and therapeutic strategies of HF.

\section{Methods}

A questionnaire which is consisted of 32 questions about current diagnostic and therapeutic strategies of HF and arrhythmia was used to evaluate awareness of physicians who are interested in HF and arrhythmia. The questionnaire has been sent to physicians by e-mail and they answered the questions online. At the end of 3 months the questionnaire was finalize and answers were collected. The Statistical Package for the Social Sciences software (IBM SPSS, version 21.0, SPSS Inc., Chicago, IL, USA) was used all statistical calculations.

\section{Results}

The study groups were consisted of 177 physicians ( 163 cardiologist, 7 cardiovascular surgeons, 6 internal medicine physicians and 1 primary care physician). Most of them have worked as a physicians for 510 years ( $\mathrm{N}: 103,58.2 \%)$. There were 55 professor and associated professor (38\%) and 122 assistant doctor and specialist (62\%). Fifty two point five percent of physicians have been worked in state hospital and 33.9\% in university hospital. Less than half of the physicians ( $\mathrm{N}: 71,40.1 \%$ ) have used natriuretic peptides in the diagnosis of HF and $76.3 \%$ of physicians were aware of HF with mid-range EF (HFmrEF) - patients with $\mathrm{HF}$ and a left ventricular ejection fraction (LVEF) that ranges from 40 to $49 \%$. Only $5.6 \%$ of physicians were able to achieve the guidelines recommended target dose of angiotensin converting enzyme inhibitors (ACEIs) and angiotensin receptor blockers (ARBs) in more than $70 \%$ of patients with HF and reduced LVEF (HFrEF). Most of the physicians (69\%) were only able to reach the treatment goals in the $41-50 \%$ of HFrEF patients. The guideline recommended treatment goals of beta blockers (BB) were achieved in $>70 \%$ patients by $5.1 \%$ of physicians. More than half of the physicians (55.9\%) achieved the treatment goals in $<30 \%$ of patients. Despite optimal medical therapy with diuretic, ACEIs, ARBs, BB and mineralocorticoid receptor antagonists (MRAs), the ratio of physicians who have $>50 \%$ symptomatic patient population were $6.8 \%$. Twenty seven point one of the physicians (27.1\%) had symptomatic patients $<25 \%$. In a symptomatic patient despite optimal medical therapy with diuretics, ACEIs/ARBs, BB, MRAs and with sinus rhythm, QRS duration less than $120 \mathrm{msn}$ on surface electrocardiogram (ECG), the $83.6 \%$ of physicians selected ivabradin therapy, $11.9 \%$ digoksin therapy and $4.5 \%$ left ventricular assist device (LVAD) or heart transplantation. In this patient, angiotensin receptor neprilysin inhibitor (ARNI) was the treatment choice among $62.7 \%$ of physicians.
Interestingly, $10.2 \%$ of physicians have never heard before ARNI. Digoksin therapy was used $<20 \%$ of patients with sinus rhythm on ECG among $76 \%$ of physicians. At patients with spontaneous echo contrast on echocardiography, $49.7 \%$ of physicians have selected antiplatelet therapy, $17.5 \%$ warfarin plus antiplatelet therapy and $31.6 \%$ no therapy. Among patients with stable angina pectoris, sinus rhythm, under maximum tolerated BB therapy, treatment choice was revascularization among $37.3 \%$ of physicians, trimetazidine among $8.5 \%$, ivabradin among $16.9 \%$, ranolazine among 12.4 and long actin nitrates among $24.9 \%$. Most of the physicians (90.4\%) have suggested searching for viability before revascularization. Carvedilol was the most chosen BB in HF patients by physicians (59.9\%). Respectively, metoprolol succinate (32.8\%), nebivolol and bisoprolol (3.4\%) and propranolol (0.6\%) were the preferred BB. Eighty three percent (83\%) of participant center had the possibility of continuous positive airway pressure for non-invasive ventilation. Only $11.3 \%$ of diabetic patients were followed by cardiologists, the rest of diabetic patients (88.7\%) were referred to endocrinologist. Fifty one (28.8\%) patients suggested their patients ICD therapy independently of heart failure etiology, if LVEF is $\leq 40 \%$. Fourty-five patients $(25.4 \%)$ considered ICD therapy for their patients, if the heart failure is ischemic origin. Only small amount of patients (11.3\%) considered the hypervolemia responsible for hyponatremia $>30 \%$ of patients. Left ventricular assist device (LVAD) and intra-aortic balloon pump (IABP) were achievable in 117 participant center (66.1\%). When the patients were discharged from hospital, symptomatic improvement in $96 \%$ of patients, decrease of natriuretic peptides (BNP and NT pro-BNP) in $18.6 \%$ of patients, euvolemic status in $73.4 \%$ of patients as a treatment success (or surrogate). Diuretic infusion was the treatment choice in the $67.2 \%$ of patients and iv. bolus diuretic was chosen in $32.8 \%$ of patients. Seventy four percent (74\%) of physicians used low dose dopamine with iv. diuretic to increase urine output in their patients. Only $9.6 \%$ of patients used the levosimendan as an inotropic support therapy in $>10 \%$ their heart failure patients. Eighty one percent of physicians (81.4\%) said that they would have chosen cardiac resynchronization therapy (CRT) if a pacemaker was necessary in a heart failure patient. We asked the physicians if they had a patient with HFrEF, NYHA II and systolic blood pressure lower than $90 \mathrm{~mm} \mathrm{Hg}$, which therapy would they have consider? The answer was low dose ACEIs and BB simultaneously in $45.8 \%$, only BB in $26.6 \%$, only ACEIs in $20.3 \%$, no therapy in $6.8 \%$ and no idea in $0.5 \%$. Sixty one (61\%) physician agreed to implant CRT-D when CRT is indicated. In a patient with acute decompensated heart failure (ADHF), pulmonary congestion and $120 \mathrm{~mm} \mathrm{Hg} \mathrm{SBP,} \mathrm{iv.} \mathrm{di-}$ uretic and vasodilatator therapy was the most preferred choice (58.8\%). Most of the physicians (70.1\%) did not prefer to give n-3 PUFA to their HF patients and. In a patient NYHA IV and QRS duration $118 \mathrm{msn}$, most of physicians (53.1\%) did not consider ICD If the patient is not a candidate for LVAD or heart transplantation. While only $13.6 \%$ physicians had $>30 \%$ patients with HFpEF, most of the physicians $(30.5 \%)$ had between $21 \%$ and $30 \%$ patients with HFpEF. In a patient on BB and ivabradin therapy if the heart rate is decreased under $55 \mathrm{bpm}, 55.9 \%$ of physician answer was to stop ivabradin therapy, $25.4 \%$ was cessation of ivabradin therapy, $4.5 \%$ was cessation of BB therapy, $10.7 \%$ was decreasing BB dosage. Only one quarter of participant center (27.7\%) had heart failure policlinic.

\section{Discussion}

Heart failure (HF) is one of the most important causes of morbidity and mortality in the world. ${ }^{1}$ It is a big burden to healthcare economy. Currently approximately 26 million adults live with HF in the world ant this number is substantially increasing with aging population. The prevalence is not clearly known in Turkey, but in the HAPPY study it was estimated as $2.9 \%$ in adults. ${ }^{4}$

In this questionnaire we aimed to evaluate awareness of physicians who are interested in HF and arrhythmias about current diagnostic and therapeutic strategies of HF. Most of the physicians (92,1\%) was 


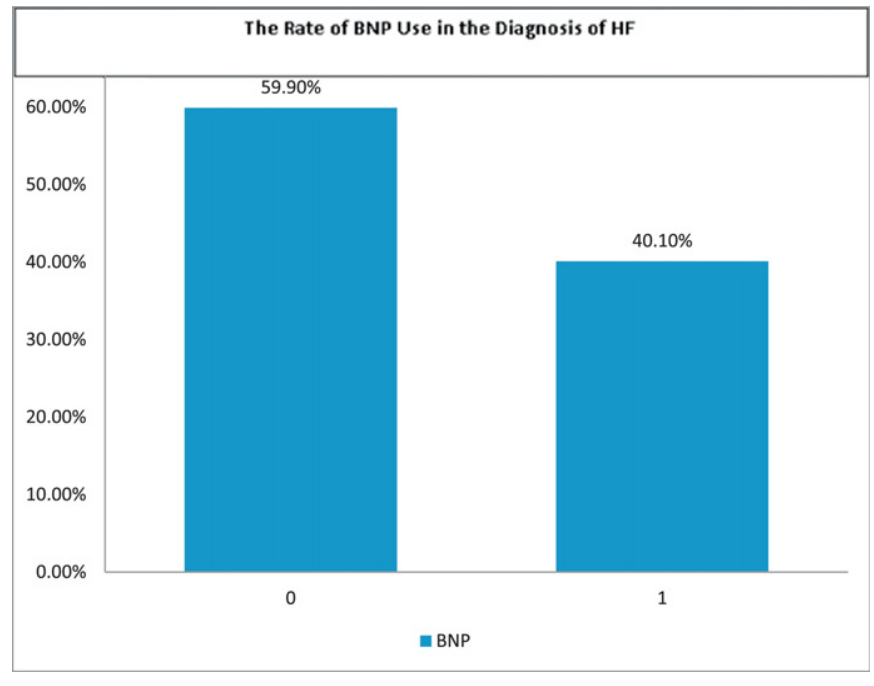

Fig. 1. The rate of BNP use in the diagnosis of HF.

working in an university hospital (3th grade health care center in Turkey) and there were much more academician (cardiologist, associated professor and professor). Natriuretic peptides (BNP and NT-proBNP) were more pronounced in the diagnosis of HF in recent European Society of Cardiology (ESC) HF guidelines (especially in the diagnosis of HFmrEF and HFpEF). ${ }^{1}$ Less than half of the physicians (40.1\%) stated to use natriuretic peptides in the diagnosis of HF (Fig. 1). 76.3\% of physicians were aware of HF with mid-range EF (HFmrEF) - patients with $\mathrm{HF}$ and a left ventricular ejection fraction (LVEF) that ranges from 40 to $49 \%$ and agreed that it was necessary to classify HF patients as HFrEF, HFmrEF and HFpEF according to LVEF. Unfortunately only $5.6 \%$ of physicians were able to achieve the guidelines recommended target dose of angiotensin converting enzyme inhibitors (ACEIs) and angiotensin receptor blockers (ARBs) in more than $70 \%$ of patients with $\mathrm{HF}$ and reduced LVEF (HFrEF). Most of the physicians (69\%) were only able to reach the treatment goals in the $41-50 \%$ of HFrEF patients. Same as ACEIs, the success rate of achievement guideline recommended BB therapy was disappointing. Only $5.1 \%$ of patients were able to achieve the treatment target $>70 \%$ of HF patients. Despite optimal medical therapy

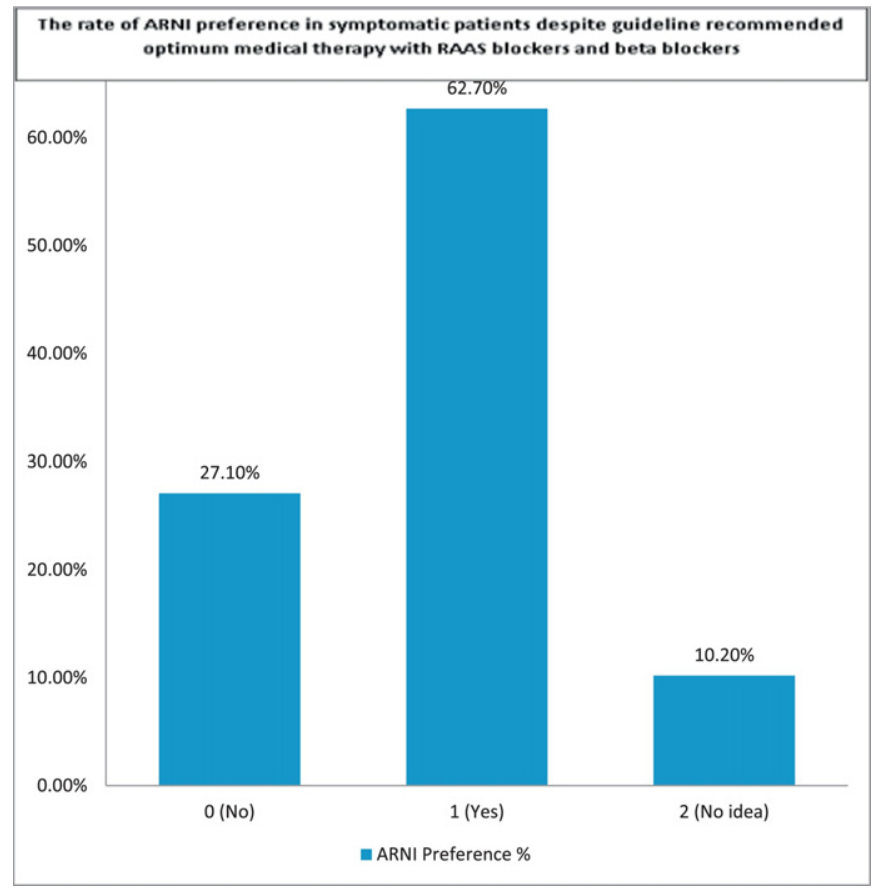

Fig. 3. The rate of ARNI preference in symptomatic patients despite guideline recommended optimum medical therapy with RAAS blockers and beta blockers.

with diuretic, ACEIs, ARBs, BB and mineralocorticoid receptor antagonists (MRAs), the ratio of physicians who have more than symptomatic patient population were $6.8 \%$ (Fig. 2). In a symptomatic patient despite optimal medical therapy with RAAS blocker and BB, most of the patients have chosen ivabradin (83.6\%) or ARNI (62.7\%). It shows physicians who is interested in heart failure, follows the suggestion of recent HF guidelines in daily practice. Interestingly, $10.2 \%$ of physicians have never heard before ARNI (Fig. 3, group 2). In a stable angina patient, revascularization was the most preferred treatment if the angina still continues despite optimal medical therapy. Carvedilol was the most preferred BB in HF patients same as daily practice. In an asymptomatic HF patient with LVEF $40 \%$, most of the patients did not consider ICD

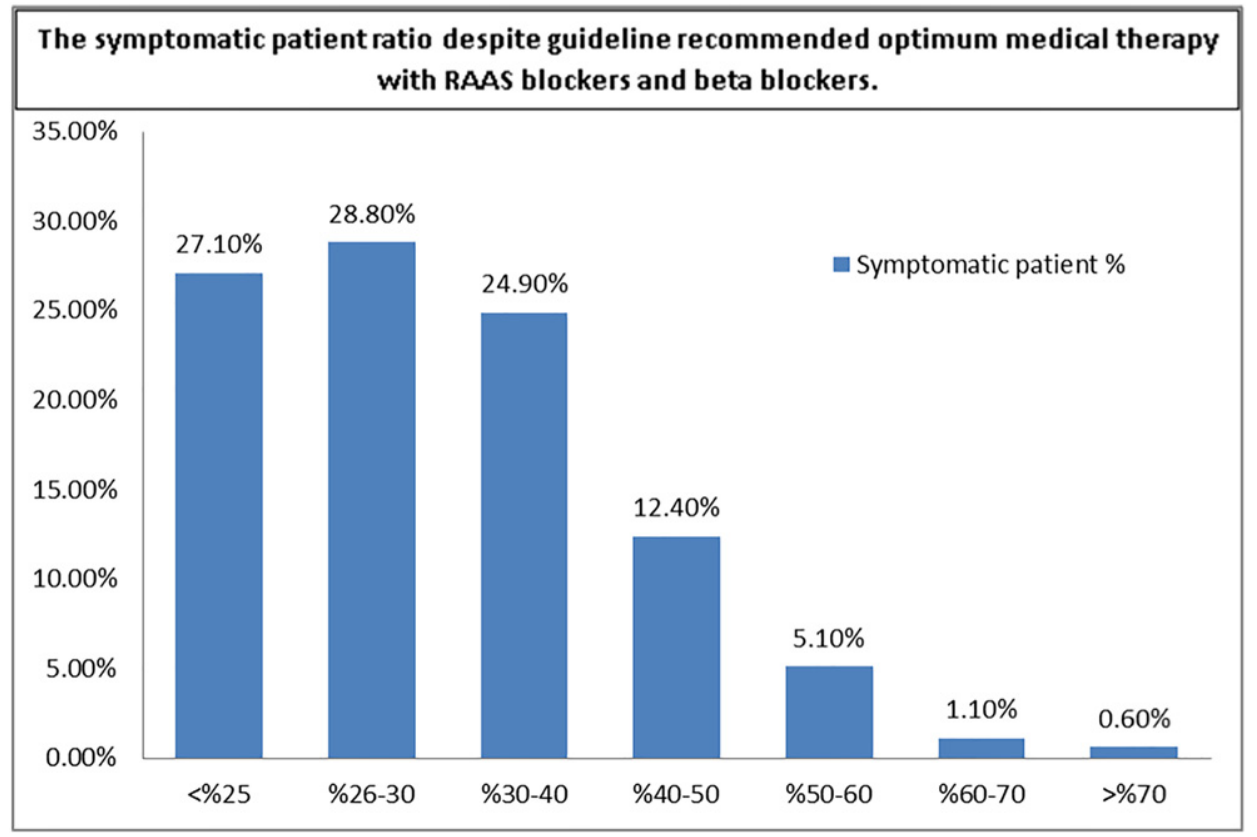

Fig. 2. The symptomatic patient rate despite guideline recommended optimum medical therapy with RAAS blockers and beta blockers. 
therapy. LVAD and IABP were achievable in the most center. Most of the patients considered symptomatic improvement and euvolemia as a treatment target. Natriuretic peptides were the treatment target by only small amount of physicians (18.6\%). Diuretic infusion was more preferred than iv. diuretic bolus in HF patients. This might be associated with the nature and stage of HF. If CRT is indicated most of the patients considered to implant CRT-D. Current ESC guideline on the management of HF recommends ACEIs or ARBs with BB at the same time for initiation therapy and to increase evidence-based maximum tolerated dose. The participant physicians have internalized this treatment strategy. N-3 PUFA treatment was not a highly preferred HF treatment. Current ESC guideline does not recommend ICD therapy to NYHA IV patients if they are not candidate for LVAD or heart transplantation. Most of the participants paid attention this recommendation. When we asked the proportion of HFpEF in their daily practice, $30.5 \%$ of participants emphasized that only small amount of heart failure patients in daily practice were HFpEF patients (13.6\%). Only one quarter of participant center (27.7\%) had heart failure policlinic.

In this questionnaire we wanted to see a picture from daily practice of physicians who are interested in heart failure. We aimed to measure awareness about current ESC heart failure guideline recommendations, new classification of HF according to LVEF and new diagnostic and therapeutic improvements.

\section{Conflict of interest}

On behalf of all authors, the corresponding author states that there is no conflict of interest.

\section{References}

1. Ponikowski P, Voors AA, Anker SD, et al. ESC guidelines for the diagnosis and treatment of acute and chronic heart failure. The task force for the diagnosis and treatment of acute and chronic heart failure of the European Society of Cardiology (ESC). Eur Heart J 2016;37:2129-2200.

2. Cowie MR, Mosterd A, Wood DA, et al. The epidemiology of heart failure. Eur Heart J 1997; 18:208-225.

3. Lloyd-Jones D, Adams RJ, Brown TM, et al. Heart disease and stroke statistics-2010 update: a report from the American Heart Association. Circulation 2010;121:e46-e215.

4. Değertekin M, Erol C, Ergene O, et al. Heart failure prevalence and predictors in Turkey: HAPPY study. Turk Kardiyol Dern Ars 2012;40:298-308. 